\title{
Democracy and Parliamentarism in Russia: Roots, Traditions, Modernity
}

\author{
Nikolaev Andrey Mikhailovich
}

Prudnikov Mikhail Nikolaevich

Churilov Sergey Nikiforovich

Ilyakov Dmitriy Vasil'evich

Glazkova Lilia Vladimirovna

Yastrebova Anna Ivanovna

Grudinin Nikita Sergeevich

Russian state social university, Moscow, 129226, Russia

Email: kafedra507@mail.ru

\section{Doi:10.5901/mjss.2015.v6n3s2p82}

\section{Annotation}

The article discusses traditions and modern state of parliamentarism in Russia. It analyses present day trends and problems of functioning of Parliament of the Russian Federation. The fact is pointed that Federation Council and State Duma as chambers of parliament of the Russian Federation didn't gain a necessary authority in the eyes of Russian citizens. One of the main reasons of that is an absence of stable order to form chambers of Federation Assembly of the Russian Federation. It is presented that finding that order is a necessary condition for a successful development of democracy institutions and parliamentarism in Russia in the future.

Keywords: Democracy; Parliamentarism; State Duma and Federation Council.

\section{Introduzione}

La nascita degli istituti parlamentari in Russia come in altri paesi del mondo (Regno Unito, Francia, Germania, Italia) ha una storia profonda. Tuttavia, in Russia il funzionamento degli organi rappresentativi del potere è stato in dipendenza diretta dalla tradizione autoritaria che caratterizza la Russia per la maggior parte della storia. La situazione è cominciata a cambiare solo negli ultimi anni quando, dopo l'adozione della Costituzione della Federazione Russa - anno 1993, in cui sono state affermate idee di democrazia, stato costituzionale, priorità dei diritti e delle libertà dell' uomo e del cittadino come valori supremi, è diventato possibile il funzionamento del principio della separazione dei poteri e del'istituto del parlamentarismo.

\section{La Teoria}

Secondo la maggior parte degli studiosi del fenomeno del parlamentarismo, il parlamento moderno ha duplice natura giuridica che combina sostanzialmente i caratteri dell'ente pubblico e quelli della rappresentazione delle opinioni politiche e degli interessi popolari. Alcuni prototipi del parlamento in Russia possono essere considerati: la Vece, lo Zemskij sobor, la Duma dell' Impero Russo, il sistema dei Consigli. Tali istituti hanno definito le caratteristiche del parlamentarismo russo: nazione, elezione, adozione di leggi, partecipazione alla gestione degli affari di Stato. L' origine del parlamentarismo russo si puo trovare nello spirito stesso del popolo russo, nelle tradizioni della sua statualità.

Ai sensi dell' art. 94 della Costituzione della Federazione Russa L'Assemblea Federale - il Parlamento della Federazione Russa - è l'organo rappresentativo e legislativo della Russia. Nella Federazione russa si realizza il modello bicamerale del parlamento: L'Assemblea Federale si compone di due camere - il Consiglio della Federazione Russa e la Duma di Stato della Federazione Russa.

Il Consiglio della Federazione è l'ente rappresentativo del potere statale con doppia natura. Da un lato, essendo 
parte integrante del parlamento federale, il Consiglio della Federazione deve funzionare nell' interesse di tutti cittadini della Russia. Dall'altro lato, il Consiglio della Federazione è l'organo che è stato fatto per proteggere gli interessi delle regioni specifiche. Ai sensi della parte 3 dell'art.95 e dell'art.96 della Costituzione della Federazione Russa La Duma dello Stato si compone di 450 deputati e viene eletta con un mandato di 5 anni.

Va notato che, frequenti cambiamenti dell'ordine di formazione del Consiglio della Federazione e la modifica permanente del sistema elettorale, con la quale si determinano i risultati delle elezioni di deputati della Duma dello Stato, sono diventati una tradizione nel moderno parlamentarismo russo. L'assenza di un ordine stabile di acquisizione del parlamento della Federazione Russa è una delle ragioni del suo basso livello nel sistema degli organi di potere statale e anche agli occhi dei cittadini russi.

\section{I Risultati}

La stabilità del funzionamento del parlamento della Federazione Russa è correlata all' ordine stabile della sua formazione. Noi riteniamo che il metodo migliore per la formazione del Consiglio sia l'elezione dei suoi membri da parte del popolo, attraverso elezioni dirette con la successiva approvazione da parte degli enti rappresentativi ed esecutivi dell'autorita pubblica dei soggetti della Federazione Russa. La procedura elettiva per l'acquisizione del Consiglio permette di mantenere il suo status di camera delle regioni e contemporaneamente lo avvicina agli abitanti delle regioni specifiche.

Allo scopo di aumentare l'efficienza della Duma di Stato, la completa realizzazione di una istituzione costituzionale di sovranità del popolo russo, la stabilizzazione della legislazione elettorale nazionale, appare necessaria l'adozione di un emendamento alla Costituzione della Federazione Russa che dovrebbe fissare i principi fondamentali del sistema elettorale dei deputati della Duma di Stato. La Parte 3 dell art.95 della Costituzione della Federazione Russa andrebbe letta come segue: "La Duma di Stato è formata da 450 deputati eletti sulla base del sistema elettorale maggioritarioproporzionale : la metà dei deputati va eletta in circoscrizioni con mandato al singolo mentre l'altra metà - in base alla lista federale dei candidati presentata alle elezioni da partiti politici e blocchi elettorali".

\section{Conclusione}

Il parlamentarismo in Russia ha affrontato un lungo e difficile percorso di sviluppo. Questo fatto è spiegato dalla tradizione autoritaria che ha prevalso in Russia per molti secoli. II parlamento moderno della Federazione Russa sta cominciando a conquistare la fiducia dei cittadini.

La procedura per la formazione del Consiglio della Federazione e il sistema elettorale dei deputati della Duma di Stato riflettono tutta l'esperienza positiva acquisita durante il funzionamento del parlamentarismo in Russia. Ciò nonostante, oggi come di consueto, i cittadini russi non ritengono che gli organi rappresentativi esistenti funzionino in modo efficace e neanche che i deputati siano "rappresentanti del popolo" ideali. Questo fenomeno mostra che sia la procedura legislativa della formazione del Consiglio della Federazione che le elezioni dei deputati della Duma di Stato sono ancora in evoluzione e hanno bisogno di una ulteriore revisione.

La qualità del funzionamento delle istituzioni parlamentari in un paese determina direttamente l'efficacia della sua modernizzazione politica nelle condizioni moderne. Incrementare la rappresentanza del popolo nell'Assemblea Federale (nel parlamento della Federazione Russa) significa avvicinare lo Stato ad un ideale democratico in cui il popolo sia l'unica fonte e il supremo sostenitore del potere sovrano dello Stato.

\section{Riferimenti}

Belonovskiy V.N. II diritto elettorale della Federazione Russa. M.: RSUH, 2010.

Belyaev I.D. Lo Zemskij Sobor in Russia. M.: Libreria di A.D. Stupin, 1902.

Biktagirov R.F., Fomin A.A., Shapiev S.M. Elezioni di deputati della Duma di Stato dell'Assemblea Federale della Federazione Russa (revisione della legislazione più recente). M.: RCOIT, 2007.

Grankin I.V. II parlamento della Russia. M.: Editore S.p.A. "Consult-banchiere", 1999.

E. Lakeman, J.D. Lambert, La ricerca dei sistemi elettorali maggioritari e proporzionali / a cura di A.S. Shugaev. M.: La casa editrice della letteratura straniera, 1958.

Lukyanov A.I. II parlamentarismo in Russia (questioni di storia, teoria e pratica): il corso di lezioni. M. : Norma: INFRA-M, 2010.

La democrazia russa: lo sviluppo, le tendenze moderne e controversie / a cura di A.V. Ivanchenko. M.: Fondo "La missione liberale", 2003.

I sistemi elettorali moderni. Edizione 2: Argentina, Germania, Svezia / A.G. Orlov, Y.I. Leibo, A.I. Rakitskaya; editore scientifico Y.A. Vedeneev, V.I. Lysenko. M.: RCOIT: Norma, 2007. 